\title{
Fluorescence study on site-specific binding of perfluoroalkyl acids to human serum albumin
}

\author{
Yan-Min Chen $\cdot$ Liang-Hong Guo
}

Received: 26 March 2008/Accepted: 2 September 2008/Published online: 15 October 2008

(C) Springer-Verlag 2008

\begin{abstract}
Binding of five perfluoroalkyl acids with human serum albumin (HSA) was investigated by sitespecific fluorescence. Intrinsic fluorescence of tryptophan214 in HSA was monitored upon addition of the chemicals. Although perfluorobutyl acid (PFBA) and perfluorobutane sulfonate (PFBS) did not cause fluorescence change, perfluorooctanoic acid (PFOA), perfluorooctane sulfonate (PFOS), and perfluorododecanoic acid (PFDoA) induced fluorescence quenching, from which binding constant of $2.7 \times 10^{5} \mathrm{M}^{-1}$ for PFOA and $2.2 \times 10^{4} \mathrm{M}^{-1}$ for PFOS was calculated. Two fluorescent probes, dansylamide (DA) and dansyl-L-proline (DP), were employed in fluorescence displacement measurements to study the interaction at two Sudlow's binding sites. At Site I, both PFBA and PFBS displaced DA with binding constants of $1.0 \times 10^{6} \mathrm{M}^{-1}$ and $2.2 \times 10^{6} \mathrm{M}^{-1}$. At Site II, PFBS and PFDoA displaced DP with binding constants of $6.5 \times 10^{6} \mathrm{M}^{-1}$ and $1.2 \times 10^{6} \mathrm{M}^{-1}$, whereas PFBA did not bind. The data were compared with fatty acids to evaluate the potential toxicological effect of these environmental chemicals.
\end{abstract}

Keywords Albumin · PFOS · PFOA · Fluorescence · Fatty acid

Electronic supplementary material The online version of this article (doi:10.1007/s00204-008-0359-x) contains supplementary material, which is available to authorized users.

Y.-M. Chen · L.-H. Guo $(\square)$

State Key Laboratory of Environmental Chemistry and Ecotoxicology, Research Center for Eco-Environmental

Sciences, Chinese Academy of Sciences, P.O. Box 2871,

18 Shuangqing Road, 100085 Beijing, China

e-mail: LHGuo@rcees.ac.cn

\section{Introduction}

Perfluorinated alkyl acids (PFAAs) have been manufactured for over 50 years. These chemicals are used in a wide variety of industrial and household applications, such as polymeric products, additives, and surfactants (3M Company 2000; LehmLer 2005). Among all the PFAAs, perfluorooctane sulfonate (PFOS) and perfluorooctanoic acid (PFOA) are the most widely utilized. These chemicals are highly stable, resistant to high temperatures, strong acids/bases, oxidizing agents, and photolysis, and thus very persistent in the environment. Broad distribution of PFAAs in humans, wildlife animals, and environment has been observed. Biomonitoring studies have shown that PFOS and PFOA are present in humans and wildlife around the world (Lehmler 2005). Although the toxicological effects of these chemicals are still under investigation, some animal studies have suggested that PFAAs affect the liver, neonatal development, the immune system, and hormone levels (Borges et al. 1990, 1992; Lau et al. 2007; Andersen et al. 2007; Roos et al. 2008). Therefore, PFAAs have been found to possess many of the characteristics of persistent organic pollutants.

Unlike polychlorinated biphenyls and dioxins, PFOS and PFOA are distributed mainly in plasma, liver, and kidney of the biologic body (Organisation for Economic Co-operation and Development (OECD) 2002). In human plasma, PFOS and PFOA were suspected to be associated with albumin (Ophaug and Singer 1980), since human serum albumin (HSA) is the most abundant protein in plasma and typically present at concentrations around $0.6 \mathrm{mM}$ (Peter 1995). As reported by Jones et al. (2003), mass spectrometry and direct in vitro binding assays were employed to investigate binding of PFAAs with serum proteins from different sources. It was found that PFOS had 
a weak affinity for carp serum steroid-binding proteins and could not displace hormones from the proteins at low concentrations. In addition, PFOS was found to bind strongly to bovine serum albumin at a 1:1 ratio. Based on these results as well as environmental and human exposure concentrations of PFOS, it was concluded that the chemical would not displace hormones from serum proteins. In another report by Han et al. (2003), binding of PFOA to rat and human plasma proteins were investigated. From sizeexclusion and ligand-blotting experiments, it was found that PFOA bound primarily to serum albumin in plasma. Based on NMR and microdesalting experiments, the dissociation constant and number of binding sites for binding with rat serum albumin and HSA were estimated to be $0.3-$ $0.4 \mathrm{mM}$ and $6-9$, respectively. The two studies described earlier confirmed the binding of PFAAs with serum albumin.

The physiological role of HSA is to transport long-chain fatty acids in blood. Curry's group reported high-resolution X-ray crystal structure of HSA cocrystallized with fatty acids, which identified at least seven binding sites for longchain fatty acids (Curry et al. 1998; Bhattacharya et al. 2000; Petitpas et al. 2001). NMR studies by Hamilton's group reveal that, among the seven fatty acid binding sites, sites 2, 4, and 5 are high affinity sites, while sites 1, 3, 6, and 7 exhibit low affinity for fatty acids (Simard et al. 2006). HSA also binds a large number of exogenous compounds such as drugs, amino acids, steroids, and metals (Pardridge 1987; Kragh-Hansen et al. 2002; Peters 1996). As first reported by Sudlow et al. (1975, 1976), HSA has two high-affinity drug binding sites, the so-called Site I and Site II. Site I is located in subdomain IIA and overlaps with fatty acid binding site 7 , while Site II is located in subdomain IIIA and overlaps with fatty acid binding sites 3 and 4 (Ghuman et al. 2005). Given the large number of fatty acid and drug binding sites on HSA, it is necessary to know if a PFAA binds at the same sites as fatty acids and to know the binding affinity at those sites, before an assessment can be made about its ability to displace fatty acids from the protein. Data obtained in the two previous studies (Han et al. 2003; Jones et al. 2003) are on the overall binding interaction, not site-specific.

In addition to X-ray crystallography and NMR, a number of other techniques have been developed and employed in the study of drug-HSA interactions, such as ultrafiltration, equilibrium dialysis, calorimetry, circular dichroism, and fluorescence (Lee and McMenamy 1980; Ashbrook et al. 1972, 1975; Kragh-Hansen 1991; Tillement et al. 1974; Hansen et al. 2001; Mahesha et al. 2006). Among them, fluorescence provides a very high signal-to-noise ratio and is exquisitely sensitive to the immediate environment of the fluorophore (Royer 2006). In previous work, we used site-specific fluorescence probes in the investigation of fatty acid binding with HSA. The results were found to be comparable to those obtained with the established methods. In this work, the same approach has been employed to study the binding of five PFAAs to HSA at three distinct sites on the protein.

\section{Experimental}

HSA (fraction V, essentially fatty acid-free, $\geq 96 \%$ purity), perfluorobutyl acid (PFBA, purity 98\%), perfluorobutane sulfonate (PFBS, purity 97\%), PFOA (purity 96\%), PFOS (purity 98\%), perfluorododecanoic acid (PFDoA, purity 95\%), dansylamide (DA), and dansyl-L-proline (DP) were all purchased from Sigma-Aldrich Co. (St Louis, MO). The protein was dissolved in $100 \mathrm{mM} \mathrm{NaH} \mathrm{PO}_{4} / \mathrm{Na}_{2} \mathrm{HPO}_{4}, \mathrm{pH}$ 7.4 , at a concentration of $100 \mu \mathrm{M}$, as determined by UVvisible spectrophotometry with an extinction coefficient $\left(E_{1}^{1 \%} \mathrm{~cm}\right)$ of 5.3 at $280 \mathrm{~nm}$, and stored at $4{ }^{\circ} \mathrm{C}$. Stock solutions of PFBA, PFBS, PFOA, and PFDoA were prepared at a concentration of $20,5,2.5$, and $10 \mathrm{mM}$, respectively, in the phosphate buffer. Stock solutions of PFOS were prepared at a concentration of $200 \mathrm{mM}$ in DMSO and diluted to $20 \mathrm{mM}$ with the phosphate buffer. Stock solutions of DA and DP $(13 \mathrm{mM})$ were prepared in acetonitrile and stored in dark.

Fluorescence emission spectra were measured on a Perkin-Elmer LS55 luminescence spectrometer (Waltham, MA, USA). Tryptophan fluorescence of HSA was initiated by exciting the protein solution at $295 \mathrm{~nm}$, which was used to minimize the contribution from tyrosine residues. Emission spectra were recorded from 300 to $500 \mathrm{~nm}$ after stepwise addition of the PFAAs. The excitation and emission slit width were both set to $15 \mathrm{~nm}$. Fluorescence displacement measurements with the two site-specific indicators DA and DP were performed by varying the perfluoro-chemical concentration while keeping both the protein and indicator concentration constant at $0.1 \mathrm{mM}$. An excitation wavelength of $350 \mathrm{~nm}$ was used for DA and $375 \mathrm{~nm}$ for DP. The emission spectra were recorded from 400 to $600 \mathrm{~nm}$. Both the excitation and emission slit width were set at $15 \mathrm{~nm}$. All the fluorescence spectra are provided in the Supporting Information.

\section{Results and discussion}

Tryptophan intrinsic fluorescence

HSA has a single tryptophan residue $\left(\operatorname{Trp}^{214}\right)$ located in the hydrophobic cavity of the IIA subdomain in fatty acid binding site 7, corresponding to the so-called Sudlow's drug binding site I region (Brown 1977). The intrinsic 
fluorescence of the unique tryptophan is very sensitive to the environment around the amino acid residue. Binding of small molecules with HSA sometimes induces changes in protein conformation and consequently its fluorescence. Therefore, $\operatorname{Trp}^{214}$ fluorescence has been employed frequently in the study of HSA interaction with fatty acids, drugs, and other exogenous chemicals (Nanda et al. 2007; Rajaian et al. 1997; Zia and Price 1976; Romanini et al. 1998).

Binding interaction of the five PFAAs to HSA was investigated by monitoring $\operatorname{Trp}^{214}$ fluorescence in the titration experiment. As can been seen in Fig. 1, addition of PFOS into HSA resulted in substantial change of $\operatorname{Trp}^{214}$ fluorescence. As PFOS concentration was increased from 0 to $3 \mathrm{mM}$, fluorescence decreased gradually. At $3 \mathrm{mM}$, the intensity was reduced to about $40 \%$ of the initial value, and became steady at higher concentrations. Concomitantly, the emission peak wavelength blue-shifted from 341 to $331 \mathrm{~nm}$, which is indicative of increased hydrophobicity around tryptophan (Lakowicz 1983). The drop in tryptophan fluorescence can be rationalized by assuming conformational change of the protein after PFOS binding and quenching by other amino acid residues, which are brought closer to tryptophan after the conformational change. Using Scatchard plot (Scatchard and Ann 1949) shown in the inset of Fig. 1, the binding constant and binding ratio of PFOS to HSA are calculated to be $2.2 \times 10^{4} \mathrm{M}^{-1}$ and 14 , respectively.

The result of PFOA is similar to PFOS (Fig. S1 in the Supporting Information). In the concentration range of 0-0.8 $\mathrm{mM}$, tryptophan fluorescence dropped progressively as PFOA was added into the protein solution, and its wavelength blue-shifted from 341 to $330 \mathrm{~nm}$. Above $0.8 \mathrm{mM}$, both the intensity and emission wavelength became steady. Using the same analysis, the binding constant and binding

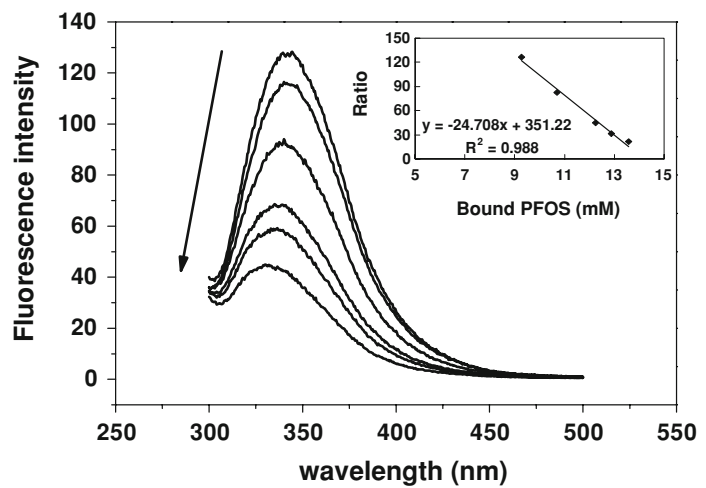

Fig. 1 Tryptophan fluorescence emission spectra of $0.1 \mathrm{mM}$ HSA in $100 \mathrm{mM}$ phosphate, $\mathrm{pH} 7.4$, as a function of PFOS concentration $(0$, 0.4, 0.7, 1.5, 2, $4 \mathrm{mM}$ ). Excitation wavelength: $295 \mathrm{~nm}$. The arrow indicates increasing concentration of PFOS. Inset is Scatchard plot for the binding of PFOS to HSA; ratio $=[\mathrm{PFOS}]_{\text {bound }} /[\mathrm{PFOS}]_{\text {free }}$ ratio of PFOA to HSA are calculated to be $2.7 \times 10^{5} \mathrm{M}^{-1}$ and 5.8, respectively. The data suggest that PFOA binds to HSA 10 times stronger than PFOS at the tryptophan site.

Addition of up to $2 \mathrm{mM}$ PFDoA into HSA reduced $\operatorname{Trp}^{214}$ fluorescence to $70 \%$ of the initial value, with no obvious shift in peak position (Fig. S2). Because of its limited solubility in the phosphate buffer, PFDoA concentration could not be increased further to reach a plateau in fluorescence intensity. Therefore, binding constant was not obtained, but judging from the slope of the titration curve, binding of PFDoA to HSA is weaker than PFOS at the site under investigation. The other two chemicals, PFBA and PFBS, did not induce any change in tryptophan fluorescence, suggesting that the four-carbon PFAAs have no significant interaction with the protein at $\operatorname{Trp}^{214}$ site. All the titration experiment data are summarized in Fig. 2. The results are displayed by a plot of the normalized fluorescence $A / A_{0}$ as a function of PFAAs concentration, where $A_{0}$ is HSA tryptophan fluorescence before addition of the chemical and $A$ is the fluorescence after addition to a certain concentration.

Site I-specific fluorescence probe

As mentioned above, HSA has two high-affinity drug binding sites, the so-called Sudlow's drug Site I and Site II (Sudlow et al. 1975, 1976). It has been shown that DA and DP are two fluorescence probes, which are specific, respectively, for the two drug binding sites on HSA (Pandey et al. 1997; Jisha et al. 2006). In their free form, the two probes emit negligible fluorescence. After binding with HSA, however, the fluorescence quantum yield is increased substantially. A competitive displacement measurement can therefore be taken to study quantitatively binding interactions between small molecules and HSA,

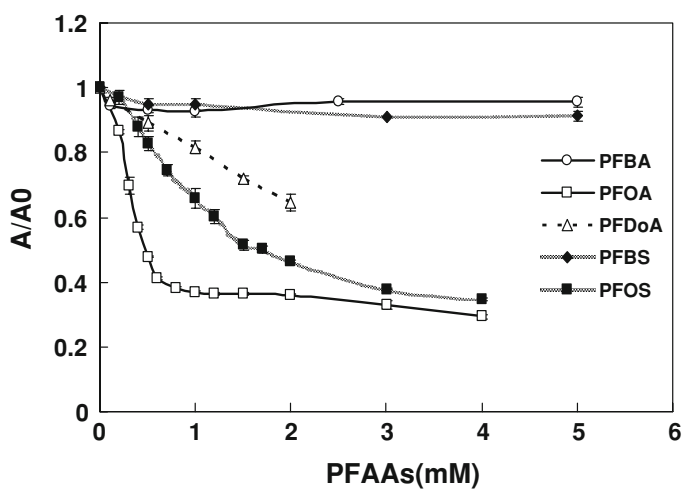

Fig. 2 Normalized tryptophan fluorescence intensity of $0.1 \mathrm{mM}$ HSA in $100 \mathrm{mM}$ phosphate, $\mathrm{pH} 7.4$, as a function of five PFAA concentrations. Excitation wavelength: $295 \mathrm{~nm}$. $A_{0}$ is the fluorescence of the probe/HSA complex before addition of PFAA, and $A$ is the fluorescence after addition of PFAAs at a certain concentration 
using either DA or DP as a signal indicator. If a compound under investigation competes with the probe for the same binding site on the protein, it would displace the probe and result in a reduction in fluorescence intensity. Quantitative information such as binding constant and binding ratio can be derived from a compound titration curve.

Competitive fluorescence measurements were carried out using $0.1 \mathrm{mM}$ HSA and $0.1 \mathrm{mM}$ DA or DP, as determined in our previous work. According to Fig. 3, titration of PFOS into HSA and DA (Site I probe) exhibited two phases of DA fluorescence change. At low PFOS concentrations $(0-0.7 \mathrm{mM})$, DA emission increased with PFOS concentration until it reached $140 \%$ of the initial intensity, along with a red-shift in peak position from 476 to $480 \mathrm{~nm}$. From 0.7 to $4 \mathrm{mM}$, fluorescence dropped, with the emission wavelength red-shift further to $483 \mathrm{~nm}$. Based on the results, it can be assumed that PFOS binds to HSA at more than one binding site, and modulates DA's fluorescence by different mechanisms. The rise in fluorescence at low PFOS concentrations is probably induced by the conformational change of the protein after PFOS binds to it at a site different from Site I, while the reduction at higher concentrations may be due to displacement of DA by PFOS at Site I. From the tryptophan fluorescence results described above, it is already known that PFOS binds to the tryptophan site with modest affinity. It will be shown later that PFOS also interacts with HSA at Site II. Therefore, it can be further postulated that the tryptophan site and Site II are where PFOS binds at low concentrations. The interaction induces protein conformation change and consequently leads to increased DA fluorescence.

In the limited concentration range examined for PFDoA, the observed signal rose, then leveled off (Figs. 4, S3). The change seems to suggest that the $\mathrm{C} 12$ perfluoro fatty acid indeed binds to HSA, but not at Site I, since it did not

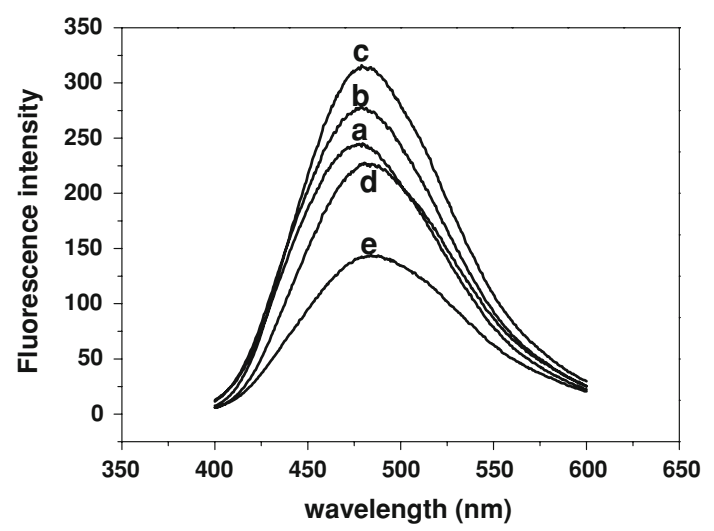

Fig. 3 Fluorescence emission spectra of $0.1 \mathrm{mM}$ DA mixed with $0.1 \mathrm{mM}$ HSA in $100 \mathrm{mM}$ phosphate, $\mathrm{pH} 7.4$, with addition of $(a) 0$, (b) $0.2,(c) 0.7,(d) 2$, and (e) $4 \mathrm{mM}$ PFOS. Excitation wavelength: $350 \mathrm{~nm}$

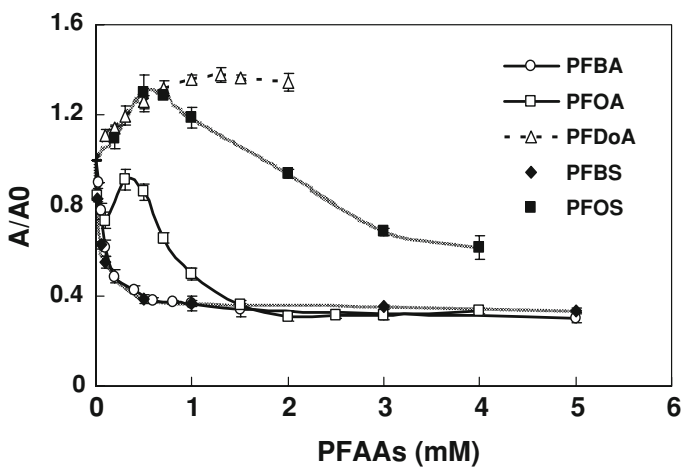

Fig. 4 Normalized fluorescence emission intensity of $0.1 \mathrm{mM}$ DA mixed with $0.1 \mathrm{mM}$ HSA in $100 \mathrm{mM}$ phosphate, $\mathrm{pH} 7.4$, as a function of five PFAA concentrations. Excitation wavelength: $350 \mathrm{~nm}$

displace any DA. Similar to PFOS, the rise in DA fluorescence can be attributed to the interaction of PFDoA with the protein at the other two binding sites. The titration curve with PFOA displayed an even more complex pattern than PFOS. With increasing PFOA concentrations, DA fluorescence first dropped, then rose, and then dropped again before it became steady (Fig. S4).

The other two PFAAs, PFBS and PFBA, exhibited a well-defined exponential fluorescence decay curve (Figs. 4, S5, S6). The curve can be interpreted by invoking a competitive binding mechanism in which the compound binds to the same site on HSA as DA, and displaces it from the protein. The fact that the fluorescence reached a plateau but did not reduce to background even at high concentrations suggests that the chemical did not completely displace the probe. From the titration curve, $\mathrm{IC}_{50}$ values are obtained, which stands for the concentration of the compound required to produce $50 \%$ inhibition of indicator binding with HSA, i.e., $50 \%$ loss of its signal. According to the relationship of binding constant with $\mathrm{IC}_{50}$ in the competitive reaction (Cheng and Prusoff 1973), the binding constants of the two $\mathrm{C} 4$ compounds at Site I are calculated to be $2.2 \times 10^{6} \mathrm{M}^{-1}$ for PFBS and $1.1 \times 10^{6} \mathrm{M}^{-1}$ for PFBA.

\section{Site II-specific fluorescence probe}

By employing DP as a Site II-specific probe, similar displacement measurements were carried out for all the five PFAAs. As shown in Fig. 5, the five chemicals displayed substantially different competition curves. While DP fluorescence essentially did not change with PFBA concentration, it decreased very rapidly with PFBS (Fig. S6) and slowly with PFDoA (Fig. S3). Using the $\mathrm{IC}_{50}$ analysis described above, binding constants are estimated to be $6.5 \times 10^{6} \mathrm{M}^{-1}$ for PFBS and $1.2 \times 10^{6} \mathrm{M}^{-1}$ for PFDoA. The titration curve with PFOS exhibited two phases of 


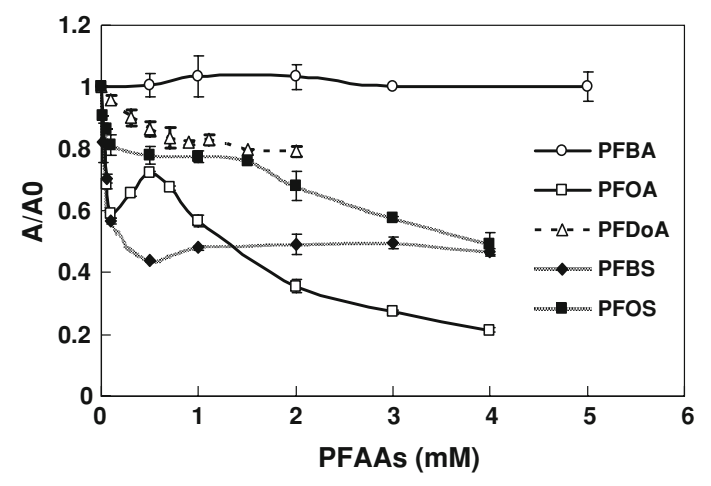

Fig. 5 Normalized fluorescence emission intensity of $0.1 \mathrm{mM}$ DP mixed with $0.1 \mathrm{mM}$ HSA in $100 \mathrm{mM}$ phosphate, $\mathrm{pH} 7.4$, as a function of five PFAA concentrations. Excitation wavelength: $375 \mathrm{~nm}$

fluorescence reduction, a rapid exponential drop followed by a more gradual decrease (Fig. S7). This suggests PFOS binds to HSA at Site II with two different affinities. Binding constant for the first phase is calculated to be $7.6 \times 10^{6} \mathrm{M}^{-1}$. Addition of PFOA resulted in multiple phases of DP fluorescence transition (Fig. S4), very similar to the curve obtained with DA probe for Site I.

Analysis and comparison with fatty acids

In this work, five PFAAs with 4,8 , and 12 carbon chains were tested, and three binding sites on HSA, the tryptophan site, and Sudlow's drug binding sites I and II were investigated separately. All the binding constants are summarized in Table 1. Although the number of chemicals tested is not large enough to generalize the results, some interesting observations can be made. Among the three binding sites, the tryptophan site has the lowest affinity $\left(10^{4}-10^{5} \mathrm{M}^{-1}\right)$, and Site II has the highest affinity (close to $10^{7} \mathrm{M}^{-1}$ ). In addition, the tryptophan site has a strong preference for long-chain chemicals, as the two PFBAs do not bind at all. The opposite seems to be the case for Site I, where only PFDoA (C12) does not bind. Site II does not have any clear preference over chain length, but it shows some exclusion for sulfonate terminal groups. Previous $\mathrm{X}$-ray crystallography work has indicated that the medium-

Table 1 Binding constants $\left(\mathrm{M}^{-1}\right)$ of five PFAAs with HSA at three specific sites

\begin{tabular}{llll}
\hline & Trp site & Site I & Site II \\
\hline PFBA & $*$ & $(1.1 \pm 0.1) \times 10^{6}$ & $*$ \\
PFBS & $*$ & $(2.2 \pm 0.1) \times 10^{6}$ & $(6.5 \pm 0.7) \times 10^{6}$ \\
PFOA & $(2.7 \pm 0.24) \times 10^{5}$ & $* *$ & $* *$ \\
PFOS & $(2.2 \pm 0.43) \times 10^{4}$ & $* *$ & $(7.6 \pm 0.3) \times 10^{6}$ \\
PFDoA & $* *$ & $*$ & $(1.2 \pm 0.04) \times 10^{6}$
\end{tabular}

* no binding, ** binding but the constant is not obtained chain fatty acids bind with HSA primarily at Site I, whereas long-chain fatty acids have a higher affinity for Site II. Examining each individual compound, PFBA shows very high site selectivity, and binds to HSA at Site I only. PFBS and PFDoA possess modest selectivity, discriminating against only the tryptophan site and Site I, respectively. Interestingly, PFOS and PFOA display no selectivity for the three binding sites on HSA. It seems that the two eight-carbon compounds have the optimal balance between chain length and polarity to fit to the three structurally different binding pockets.

In our previous work, interaction of butyric acid, octanoic acid, and dodecanoic acid with HSA at the three binding sites was studied (Chen and Guo, unpublished work). Binding constant was measured to be $1.8 \times 10^{5} \mathrm{M}^{-1}$ at Site I and $9.2 \times 10^{3} \mathrm{M}^{-1}$ at Trp-214 site for butyric acid, and $3.1 \times 10^{6} \mathrm{M}^{-1}$ at Site I, $1.1 \times 10^{7} \mathrm{M}^{-1}$ at Site II and $7.0 \times$ $10^{4} \mathrm{M}^{-1}$ at Trp-214 site for octanoic acid. Dodecanoic acid induced multiple phases of fluorescence change from which a binding constant could not be derived. Comparing the two categories of chemicals, the order of affinity for fatty acids among the three binding sites is the same as that for the PFAAs. Nonetheless, distinct differences exist between the two groups. Substitution of hydrogen atoms in fatty acids by fluorine transforms the four-carbon alkyl acids from weakly binding to nonbinding at the tryptophan site I. Similarly, the same substitution changes the 12-carbon fatty acid from high-affinity binding to nonbinding at Site I. The structural basis for this drastic change remains to be resolved.

As mentioned in the "Introduction," HSA is mainly responsible for the binding and transport of long-chain fatty acids in blood plasma. It also binds with a large variety of endogenous and exogenous chemicals such as hormones and drugs. Interaction of PFAAs with the protein has at least two foreseeable physiological effects, displacement of endogenous chemicals bound to HSA, and bioavailability of PFAAs in vivo. Based on our results, some PFAAs bind to HSA at the same site as fatty acids, with a similar affinity $\left(10^{4}-10^{6} \mathrm{M}^{-1}\right)$. Therefore, the chemical in principle has the ability to compete and displace fatty acids from the protein, and consequently change the distribution between free and bound fatty acids in plasma. Given the fact that PFAAs' concentration in human blood samples from general population is in the nanogram per milliliter range (Olsen et al. 2003a, b; Butenhoff et al. 2004), it is very unlikely that they would compete with fatty acids. However, competition is still possible with other endogenous chemicals, which bind to HSA much weaker than PFAAs, or which are present at very low concentrations. From another viewpoint, binding of PFAAs to the protein might be a positive effect, if the chemical itself is toxic. In general, a toxicant needs to be in its free state to assert its toxic effect. Interaction with HSA 
would lower the concentration of free PFAAs and reduce its bioavailability. In addition, such binding also plays an important role in the absorption, distribution, metabolism, and excretion of PFAAs in human body.

\section{Conclusion}

Noncovalent binding interaction between five PFAAs and HSA was investigated by three site-specific fluorescence probes. It was found that the chemicals bind with the protein at the same sites as fatty acids, with similar affinity $\left(10^{4}-10^{6} \mathrm{M}^{-1}\right)$. The chain length and acid head group of a PFAA have profound impact on its preference for binding sites and on its binding affinity. The data have provided some quantitative information for the future study of molecular toxicology and pharmacokinetics of these environmentally important chemicals.

Acknowledgments This work was supported by the Chinese Academy of Sciences (KZCX2-YW-420-1) and the National Natural Science Foundation of China (20621703).

\section{References}

3M Company (2000) Determination of serum half-lifes of several fluorochemicals, US Environmental Protection Agency Public Docket AR226-0645. 3M Company, St Paul

Andersen ME, Butenhoff JL et al (2007) Perfluoroalkyl acids and related chemistries - toxicokinetics and modes of action. Toxicol Sci 102:3-14

Ashbrook JD, Spector AA, Fletcher JE (1972) Medium chain fatty acid binding to human plasma albumin. J Biol Chem 247:70387042

Ashbrook JD, Spector AA, Santos EC, Fletcher JE (1975) Long chain fatty acid binding to human plasma albumin. J Biol Chem 250:2333-2338

Bhattacharya AA, Grune T, Curry S (2000) Crystallographic analysis reveals common modes of binding of medium and long-chain fatty acids to human serum albumin. J Mol Biol 303:721-732

Borges T, Glauert HP, Chen LC, Chow CK, Robertson LW (1990) Effect of the peroxisome proliferator perfluorodecanoic acid on growth and lipid metabolism in Sprague-Dawley rats fed three dietary levels of selenium. Arch Toxicol 64:26-30

Borges T, Robertson LW, Peterson RE, Glauert HP (1992) Doserelated effects of perfluorodecanoic acid on growth, feed intake and hepatic peroxisomal beta-oxidation. Arch Toxicol 66:18-22

Brown JR (1977) Serum albumin: amino acid sequence. In: Rosenoer VM, Oratz M, Rothschild MA (eds) Albumin structure, function and uses. Pergamon Press, Oxford

Butenhoff JL, Gaylor DW, Moore JA et al (2004) Characterization of risk for general population exposure to perfluorooctanoate. Regul Toxicol Pharmacol 39:363-380

Cheng YC, Prusoff WH (1973) Relationship between the inhibition constant $\left(\mathrm{K} \_\mathrm{i}\right)$ and the concentration of inhibitor which causes 50 per cent inhibition (I_(50)) of an enzymatic reaction. Biochem Pharmacol 22:3099-3108
Curry S, Mandelkow H, Brick P, Franks N (1998) Crystal structure of human serum albumin complexed with fatty acid reveals an asymmetric distribution of binding sites. Nat Struct Biol 5:827-835

Ghuman J, Zunszain PA, Petitpas I, Bhattacharya AA, Otagiri M, Curry S (2005) Structural basis of the drug-binding specificity of human serum albumin. J Mol Biol 353:38-52

Han X, Snow TA, Kemper RA, Jepson GW (2003) Binding of perfluorooctanoic acid to rat and human plasma proteins. Chem Res Toxicol 16:775-781

Hansen UK, Hellec F, de Foresta B, le Maire M, Møller JV (2001) Detergents as probes of hydrophobic binding cavities in serum albumin and other water-soluble proteins. Biophys J 80:28982911

Jisha VS, Arun KT, Hariharan M, Ramaiah D (2006) Site-selective binding and dual mode recognition of serum albumin by a squaraine dye. J Am Chem Soc 128:6024-6025

Jones PD, Hu W, de Coen W, Newsted JL, Giesy JP (2003) Binding of perfluorinated fatty acids to serum proteins. Environ Toxicol Chem 22:2639-2649

Kragh-Hansen U (1991) Octanoate binding to the indole- and benzodiazepine-binding region of human serum albumin. Biochem J 273:641-644

Kragh-Hansen U, Chuang VTG, Otagiri M (2002) Practical aspects of the ligand-binding and enzymatic properties of human serum albumin. Biol Pharm Bull 25:695-704

Lakowicz JR (1983) Principles of fluorescence spectroscopy. Plenum Press, New York

Lau C, Anitole K, Hodes C, Lai D, Pfahles-Hutchens A, Seed J (2007) Perfluoroalkyl acids: a review of monitoring and toxicological findings. Toxicol Sci 99:366-394

Lee IY, McMenamy RH (1980) Location of the medium chain fatty acid site on human serum albumin. Residues involved and relationship to the indole site. J Biol Chem 255:6121-6127

Lehmler HJ (2005) Synthesis of environmentally relevant fluorinated surfactants-a review. Chemosphere 58:1471-1496

Mahesha HG, Singh SA, Srinivasan N, Appu Rao AG (2006) A spectroscopic study of the interaction of isoflavones with human serum albumin. FEBS J 273:451-467

Nanda RK, Sarkar N, Banerjee R (2007) Probing the interaction of ellagic acid with human serum albumin: a fluorescence spectroscopic study. J Photochem Photobiol A Chem 192:152-158

Olsen GW, Burris JM, Burlew MM, Mandel JH (2003a) Epidemiologic assessment of worker serum perfluorooctanesulfonate (PFOS) and perfluorooctanoate (PFOA) concentrations and medical surveillance examinations. J Occup Environ Med $45: 260-270$

Olsen GW, Church TR, Miller JP et al (2003b) Perfluorooctanesulfonate and other fluorochemicals in the serum of American red cross adult blood donors. Environ Health Perspect 111:18921901

Ophaug RH, Singer L (1980) Metabolic handling of perfluorooctanoic acid in rats. Proc Soc Exp Biol Med 163:19-23

Organisation for Economic Co-operation and Development (OECD) (2002) Draft assessment of perfluorooctane sulfonate (PFOS) and its salt: complete assessment. Available via DIALOG. http://www.oecd.org/dataoecd/23/18/2382880.pdf. Cited 13 Oct 2005

Pandey RK, Constantine S et al (1997) Synthesis, photophysical properties, in vivo photosensitizing efficacy and human serum albumin binding properties of some novel bacteriochlorins. J Med Chem 40:2770-2779

Pardridge WM (1987) Plasma protein-mediated transport of steroid and thyroid hormones. Am J Physiol 252:157-164

Peter TJ (1995) All about albumin: biochemistry, genetics, and medical applications. Academic Press, New York 
Peters TJ (1996) All about albumin: biochemistry, genetics, and medical applications. Academic Press, San Diego

Petitpas I, Grune T, Bhattacharya AA, Curry S (2001) Crystal structures of human serum albumin complexed with monounsaturated and polyunsaturated fatty acids. J Mol Biol 314:955-960

Rajaian H, Symonds HW, Bowmer CJ (1997) Drug binding sites on chicken albumin: a comparison to human albumin. J Vet Pharmacol Ther 20:421-426

Romanini D, Avalle G, Farruggia B (1998) Spectroscopy features of the binding of polyene antibiotics to human serum albumin. Chem Biol Interact 115:247-260

Roos PH, Angerer J, Dieter H, Wilhelm M, Wolfle D, Hengstler JG (2008) Perfluorinated compounds (PFC) hit the headlines. Arch Toxicol 82:57-59

Royer CA (2006) Probing protein folding and conformational transitions with fluorescence. Chem Rev 106:1769-1784

Scatchard G, Ann NY (1949) The attractions of proteins for small molecules and ions. Acad Sci 51:660-663
Simard JR, Zunszain PA, Hamilton JA (2006) Location of high and low affinity fatty acid binding sites on human serum albumin revealed by NMR drug-competition analysis. J Mol Biol 361:336-351

Sudlow G, Birkett DJ, Wade DN (1975) The characterization of two specific drug binding sites on human serum albumin. Mol Pharmacol 11:824-832

Sudlow G, Birkett DJ, Wade DN (1976) Further characterization of specific drug binding sites on human serum albumin. Mol Pharmacol 12:1052-1061

Tillement JR, Zini R, D'athis P, Vassent G (1974) Binding of certain acidic drugs to human albumin: theoretical and practical estimation of fundamental parameters. Eur J Clin Pharmacol 7:307-313

Zia H, Price JC (1976) Binding study of tetracyclines to human serum albumin using difference spectrophotometry. J Pharm Sci 65:226-230 